\title{
Unspecified Cleavage Reagents Used
}

National Cancer Institute

\section{Source}

National Cancer Institute. Unspecified Cleavage Reagents Used. NCI Thesaurus. Code C161897.

An indication that any reagents used in the preparation of cleaved peptides for physicochemical analysis were not specified. 